\title{
Implementación de un entorno virtual para la enseñanza/aprendizaje a distancia de las Matemáticas
}

\section{Implementation of a virtual environment for distance teaching / learning of Mathematics}

\author{
Joaquín Castelló ${ }^{1}$, Carlos Galindo ${ }^{1}$, Pablo Gregori ${ }^{1}$, Vicente Martínez ${ }^{1}$ y Jorge Castañeda ${ }^{2}$ \\ castello@uji.es, galindo@uji.es,gregori@uji.es, martinez@uji.es, dirtecnoeduca@upoli.edu.ni \\ ${ }^{1}$ Departamento de Matemáticas \\ Universitat Jaume I \\ Castelló, España \\ 2 Dirección de Tecnologías Educativas \\ Universidad Politécnica de Nicaragua \\ Costado Sur Colonia Rubén Darío, Managua, \\ Nicaragua
}

\begin{abstract}
Resumen- En este trabajo detallamos como poner en práctica un Entorno Virtual de Enseñanza/Aprendizaje en el ámbito de las matemáticas. Explicamos cómo hacerlo desde sus inicios, cuando se realiza el diseño del mismo, también cómo ejecutarlo y las acciones que deberían llevarse a cabo a su finalización. Indicamos las acciones concretas que deberían implementarse teniendo en cuenta que la materia objeto del entorno virtual es la matemática. Se reconoce el importante rol que deben jugar las TICs y se indican algunas de sus funciones concretas dentro del entorno virtual. Analizamos la importancia de la tutorización virtual y el acompañamiento constante que ésta debe realizar para evitar el abandono de los estudios.
\end{abstract}

Palabras clave: entornos virtuales de aprendizaje, tecnologías de información y comunicación, matemáticas.

Abstract- In this paper we detail how to put into practice a Virtual Teaching / Learning Environment in the field of mathematics. We explain how to do it from the beginning, including the design, as well as to carry out its implementation and the actions that should be carried out when it is finished. We indicate the concrete actions that should be implemented taking into account that the subject matter of the virtual environment is mathematics. Our environment recognizes the important role that ICTs must play and indicates some of their specific functions within the virtual environment. We analyze the importance of virtual tutoring and the constant accompaniment that it must perform to avoid dropping out of studies.

Keywords: virtual learning environments, information and communication technologies, mathematics.

\section{INTRODUCCIÓN}

La educación a distancia tiene una larga historia en el mundo académico. Pero ha sido durante las últimas décadas, con los enormes avances que se han producido en el mundo de las tecnologías de la información y la comunicación, cuando las grandes universidades americanas (véase Martínez, 2017), y también europeas (véase, Gil y Roca-Piera, J 2011), han generalizado la oferta de un gran número de cursos de este tipo. Para este tipo de educación es esencial disponer de un Entorno Virtual de Enseñanza/Aprendizaje (EVEA) adecuado a la materia que queramos enseñar y aprender. La matemática es una materia difícil de enseñar, pues necesita algún soporte, no sólo verbal, en el que apoyarse. En este trabajo vamos a describir cómo proporcionar un entorno virtual que facilita enseñar y aprender matemáticas a distancia.

Para la puesta en práctica de un EVEA en formación a distancia es importante realizar algunas acciones previas. Si éstas no han sido diseñadas adecuadamente, el resto de acciones podrían no ser eficientes y obtendríamos una formación peor.

En la sección 3 de este artículo se tratarán algunas de las acciones previas antes mencionadas y se describirá cómo poner en práctica un EVEA diseñado especialmente para la enseñanza a distancia de las matemáticas.

Además, en la sección 4, proporcionaremos algunos datos sobre una experiencia piloto realizada al impartir algunas asignaturas de matemáticas de un máster de educación superior. Un EVEA con las características mencionadas fue implementado al realizar esa experiencia. Los datos se exponen en comparación con los de un curso anterior y las ventajas del EVEA son analizadas.

Finalmente, nuestro artículo contiene una sección de conclusiones en la que proponemos un análisis a largo plazo de nuestra propuesta y la realización de pequeños ajustes al mismo.

\section{CONTEXTO}

Como se ha dicho, no es posible abordar cursos de educación a distancia sin diseñar un EVEA adecuado para ello. En este trabajo se ha puesto el foco en cursos universitarios en los que se imparten contenidos matemáticos con un grado de complejidad elevado, en particular en grados de ingeniería o matemáticas. El EVEA que proponemos, por tanto, será de utilidad para el profesorado de estos títulos o materias y estará destinado a las alumnas y los alumnos de los mismos.

El objetivo de un EVEA es que los estudiantes a distancia puedan seguir su instrucción de forma sencilla, con contenidos de calidad, tutorización constante y ser evaluados de manera 
justa. Como consecuencia obtendríamos una formación (en nuestro caso, matemática) de los estudiantes, al menos, igual de buena, que en el caso de la enseñanza presencial.

Un buen EVEA debe utilizar ineludiblemente las TICs. Además, debe tener una interfaz flexible, abierta y amigable que, en nuestro caso, permita el uso del lenguaje matemático. También facilitar la comunicación, diseñando herramientas útiles para ello (objetos como pizarras virtuales, compiladores de LaTeX, etc. serán primordiales), permitir comunicaciones síncronas y asíncronas, tener herramientas y recursos TIC personalizados, incorporar espacios de interactividad y socialización y ser adecuado para la creación e intercambio de conocimiento. Finalmente, utilizar recursos y componentes que deben ser validados con objeto de asegurar un correcto funcionamiento posterior.

Para comprobar la viabilidad de nuestra propuesta hemos llevado a cabo una experiencia piloto en el Máster Universitario en Matemática Computacional de la Universitat Jaume I de Castellón (España). Se ha implementado en los dos grupos existentes, presencial y a distancia. Reforzando la labor de tutorización en el grupo a distancia, debido a que el EVEA para este grupo es el único punto de interacción entre estudiantes y profesores.

\section{DESCRIPCIÓN}

\section{A. Primeras etapas en el diseño del EVEA}

En esta sección desarrollamos algunas propuestas necesarias para la puesta en práctica de un EVEA dirigido a la enseñanza de las matemáticas. Hay que tener en cuenta que la formación que proponemos debe ser flexible, abierta, adaptada a las posibilidades de espacios y tiempos del alumnado, progresiva en los contenidos, didácticamente respetuosa con las características y condiciones del aprendizaje de éstos y actualizada. A continuación detallamos algunas pautas a seguir.

Empezamos por dar una propuesta curricular de acuerdo a la titulación objeto del programa. Esta propuesta debe ser elaborada por los departamentos o unidades académicas involucradas. Es necesario indicar cómo se generarán los cursos o materias a distancia, con qué medios se cuenta, qué plataformas se van a utilizar, quién va a ser responsable de cada curso, etc.

A continuación se abordará el diseño formativo de los cursos a distancia. La puesta en marcha de un programa formativo a distancia y virtual requiere la elaboración de materiales educativos, que deben estar disponibles en el EVEA. En base a nuestra experiencia y a la literatura existente sobre el tema (García Aretio, 2008 y Castañeda, 2016), este diseño previo requiere de:

- La exploración de la formación con la que acceden los estudiantes a los cursos a distancia.

- El reconocimiento de las potencialidades y limitaciones del alumnado a distancia.

- Saber los medios y equipos docentes disponibles.

- Entender que las características de la educación a distancia producen una nueva relación docente-estudiante $\mathrm{y}$ estudiante-estudiante.

- Tener previsto cuáles son los materiales y las destrezas mínimas necesarias para el desarrollo del curso. Esta información se ofrecerá a los estudiantes al inicio del curso.
- Establecer cuál será la metodología y los objetivos del curso, lo que permitirá indicar cómo se realizará la evaluación de los estudiantes.

- Preparar guías de trabajo por semana o por módulo, así como por temas, que orienten a los estudiantes a distancia en la distribución de tiempos y actividades que promuevan el aprendizaje autónomo y colaborativo.

- Planificar las prácticas de aprendizaje, considerando el tiempo real para su ejecución (bimestre, trimestre, cuatrimestre, semestre). Tanto las guías como las prácticas han de contar con los medios informáticos adecuados para el uso del lenguaje matemático. En este sentido se buscará el apoyo de expertos en las TIC para facilitar la edición de fórmulas, tablas, gráficas, etc. necesarias en el campo matemático.

Posteriormente se deben diseñar los materiales a distancia que se van a poner a disposición de los estudiantes. Para ello hay que determinar cuestiones como el estilo del texto de cada curso, sus recursos multimedia y el software matemático asociado. Los materiales tendrán que ser rigurosos y permitir el aprendizaje de manera autónoma. El carácter constructivo, de andamiaje, de las competencias matemáticas exige de unos materiales que permitan la autoevaluación del alumno al final de cada unidad o lección. Si no se supera la autoevaluación, el alumno debe repetir el proceso o acudir a la tutoría para alcanzar destrezas que será necesario dominar para poder acceder a los contenidos siguientes. Esta autoevaluación puede realizarse mediante un cuestionario de preguntas tipo test, para comprobar el conocimiento de definiciones, propiedades y ejemplos, así como para autoevaluar competencias procedimentales (como la aplicación de algoritmos) o actitudinales (como la elección de técnicas y su aplicación o la demostración de propiedades). Dada la complejidad de este tipo de competencias en el ámbito científico, y en particular en el matemático, el cuestionario puede devolver una retroalimentación limitada, por lo que el alumno tiene la responsabilidad de acudir a la tutoría para salvar el obstáculo. Respecto al software, recomendamos utilizar software libre, ya que rompe barreras socioeconómicas y permite que el alumnado disponga de él en su equipo personal y pueda continuar usándolo en el futuro.

Se propondrán actividades similares a las que se harían en un entorno presencial, que mantengan al alumnado comprometido con sus estudios (Martínez, 2017):

- Lección magistral: presentación de un tema lógicamente estructurado mediante la exposición verbal por parte del profesor.

- Ejercicios y problemas: desarrollo e interpretación de las soluciones obtenidas como respuesta a las cuestiones planteadas. Es un complemento de la lección magistral, además de constituir un test para comprobar la comprensión de ésta.

- Estudio de casos: análisis completo de un hecho, problema o suceso real con la finalidad de un mayor conocimiento de la materia.

- Aprendizaje por proyectos: realización de un proyecto para resolver un problema o abordar una tarea mediante la planificación, diseño y realización de una serie de actividades propuestas. El lector puede ver una implementación de esta metodología en (Pitarch et al., 2013). 
- Aula invertida (flipped classroom): metodología que consiste en transferir el trabajo pasivo de determinadas actividades fuera del aula, utilizando el tiempo de clase presencial para atender dudas, potenciar procesos de adquisición de conocimientos, realizar ejercicios y prácticas o realizar tareas de asimilación de conceptos que previamente han sido estudiados fuera del aula, etc. (véase Sein-Echaluce et al., 2015).

- Tareas complementarias: elaboración de informes, visualizar videos, exposiciones orales, seminarios, conferencias, etc.

El EVEA utilizará una interfaz flexible que permita la introducción de diversas herramientas que fomenten la interacción entre profesores y alumnos, e inter-alumnos. Uno de los puntos difíciles de las matemáticas es su lenguaje propio. La simbología que utiliza va mucho más allá del alfabeto latino y la comunicación oral de matemáticas no puede ir más allá de ideas intuitivas. La comunicación escrita precisa de editores de texto muy específicos y es mucho más costosa que la redacción de textos de otros ámbitos jurídicos o humanistas. Cuando la comunicación escrita (online) debe ser instantánea (síncrona), se precisa de TICs. Una opción es la videoconferencia en la que la cámara enfoca una pizarra convencional o al escritorio donde profesor y/o alumno representan sus preguntas y respuestas. Otra opción muy recomendable es la utilización de una pizarra virtual (como $A$ Web Whiteboard), donde profesor y alumno, pueden escribir simultáneamente desde sus ordenadores o tabletas. El funcionamiento de la interfaz del EVEA se debe poner a prueba antes de la apertura del curso. Es importante que nada falle ya que luego produce frustración en los alumnos. Sería conveniente tener ayuda profesional de personal técnico que administre la interfaz. Este personal deberá estar familiarizado con el campo matemático, para que una correcta comunicación sea posible.

Para terminar, habrá que diseñar una guía detallada de modo que los estudiantes conozcan a priori los contenidos del curso. Esta guía tiene que ser más completa que la que se ofrece a los estudiantes presenciales, pues resulta mucho más complicado seguir las materias matemáticas a distancia.

\section{B. Puesta en práctica de un EVEA}

Uno de los pilares básicos para el éxito de la implementación de un EVEA para estudiantes a distancia es la selección del profesorado más adecuado, por su preparación y compromiso con este tipo de enseñanza. Será conveniente un curso de formación específica previa y un control sobre su capacitación. Estos profesores podrían estar elegidos por el departamento o facultad en la que se integre la enseñanza. Nos parece óptimo una mezcla entre profesorado experimentado y profesorado novel, que sume a la experiencia de unos, la ilusión y los conocimientos sobre las nuevas herramientas de los otros.

Los estudiantes deben saber de la dificultad del aprendizaje de las materias matemáticas sin la guía directa de un profesor (véase Arnal et al. 2016 y 2017). Por eso, un curso de este tipo les exigirá un alto coste temporal. De ahí la necesidad de que el EVEA esté diseñado específicamente para ello. Además, los estudiantes tienen que comprometerse a seguir las enseñanzas al día. En otro caso será muy difícil sacar algún rendimiento positivo. Por ello, el profesorado debe comprometerse a realizar un acompañamiento constante del alumnado para atender sus dudas y ayudarles a resolver los posibles problemas técnicos que se les puedan presentar (véase Gregori et al., 2018).
También debe realizar un cierto control semanal o bisemanal sobre los avances obtenidos por el alumnado.

Siguiendo la guía detallada indicada en la sección anterior, se propondrán una serie de actividades que habrán sido diseñadas con anterioridad y permitirán el aprendizaje completo de cada uno de los puntos de la guía. Estas actividades contemplarán aspectos teóricos y prácticos, así como el uso de medios informáticos. Las actividades se deben orientar tanto al trabajo individual como al colectivo, además de fomentar la socialización entre los estudiantes. A esto último pueden contribuir tanto el foro de avisos del profesor, como los foros de debate, en los que los estudiantes pueden discutir y ayudarse mutuamente. Cada actividad será fácilmente evaluable para que el estudiante lleve seguimiento de su evolución a lo largo del curso. Las herramientas informáticas, que serán de uso sencillo gracias a la interfaz, tendrán que permitir, bien con fotos, vídeos, pizarras electrónicas, el uso de lenguajes técnicos como LaTeX, un fácil visionado por parte de profesores y estudiantes de todo tipo de objetos matemáticos, para simplificar la comunicación entre ellos.

De excepcional importancia, para la educación a distancia, es que el EVEA contenga un espacio de tutorías dinámico. Hay que considerar la inclusión de dos tipos herramientas para la comunicación:

- la asíncrona, como un foro donde el alumnado expone sus dudas, y el profesor las responde con brevedad, pero en otro momento.

- la síncrona, como una videoconferencia, donde se debate in situ.

La función tutorial debe saber combinar el conocimiento matemático con las condiciones particulares en las que se llevará a cabo la docencia, especialmente más compleja cuando se desarrolla en espacios virtuales y en esta ciencia. Para ello, se potenciarán foros virtuales, foros de discusión, mensajería instantánea (todo ello desde la plataforma educativa), correo electrónico, videoconferencias a través de diversas plataformas (por ejemplo, Blackboard Collaborate o Hangouts), pizarras virtuales (como $A$ Web Whiteboard, https://awwapp.com/), o edición de documentos matemáticos (Overleaf, https://www.overleaf.com/project).

Dentro de la puesta en práctica del EVEA supervisaremos la calidad de los materiales puestos a disposición del alumno. Para ello realizaremos reuniones de seguimiento del trabajo realizado por los estudiantes y de las imperfecciones detectadas por los estudiantes en los materiales disponibles. Controlaremos el tiempo necesario para que los estudiantes desarrollen los trabajos propuestos en el EVEA para que, en caso de que sea excesivo o demasiado corto, puedan mejorarse de cara al siguiente curso. Finalmente estudiaremos el nivel de dificultad que tienen las actividades propuestas. En definitiva, se trata de, con esta experiencia, poder mejorar las siguientes ediciones.

Un apartado importante en la puesta en práctica del EVEA es la evaluación de las actividades realizadas por los estudiantes. Hay que tener en cuenta que la evaluación ha de ser a distancia y que los trabajos versan sobre técnicas matemáticas. Para ello proponemos: exámenes y cuestionarios online, trabajos académicos entregables online, resolución de ejercicios y problemas usando software educativo libre, defensa pública de trabajos online, proyectos colaborativos, portafolios digitales, etc. 
Se fomentará la colaboración entre los estudiantes mediante el uso de herramientas de construcción colaborativa de conocimiento. Esta colaboración potenciará la implicación del alumno en el curso y la sensación de sentirse acompañado en su formación. Entre otras, destacamos las bases de datos online, las conferencias online o seminarios web (webinars), wikis, blogs educativos y grupos de discusión online.

Los administradores del EVEA deben recoger sistemáticamente datos del funcionamiento del mismo, y proporcionar informes sobre la calidad de la metodología. Asegurar la calidad no deberá suponer a un exceso en la burocratización de los mecanismos y los informes deben estar confeccionados de manera que no limiten la innovación educativa dentro de la Universidad.

\section{RESULTADOS}

Para tratar de decidir si las ideas anteriores producen buenos resultados educativos, hemos realizado una experiencia piloto con algunos cursos de un máster que contiene asignaturas de matemáticas. Esta experiencia se realizó en el curso 2017/18. En cursos previos, aunque había enseñanza presencial y a distancia (con pocos estudiantes), se utilizaba un EVEA mucho más primitivo.

La calidad de un EVEA vendrá dada en la medida en que las competencias previstas en el programa instruccional son alcanzadas de la misma manera que las alcanzaría una educación presencial clásica.

Un aspecto que no debe olvidarse es la evaluación docente. Todos los integrantes del proceso de enseñanza/aprendizaje (profesorado, estudiantado, gestores y administradores del EVEA) deben valorar la gestión y desempeño del mismo mediante cuestionarios. Las preguntas de los cuestionarios tienen que servir para detectar si ha habido disfunciones en el desarrollo del proceso y se hará hincapié tanto en la usabilidad de los materiales docentes como en la temporalización de las actividades. La difusión interna de los resultados de esta evaluación sirve para generar las acciones de mejora de cara a ediciones futuras. Finalmente, los contrastes de experiencias similares entre profesionales de distintas universidades son muy enriquecedoras (difusión externa), además de ampliar la perspectiva de análisis de los aspectos considerados en el EVEA propuesto. En este sentido, la asistencia a jornadas de innovación docente, y en particular de aprendizaje matemático a distancia, resultan muy positivas.

Tabla 1. Indicadores de mayor relevancia del título

\begin{tabular}{|c|c|c|}
\hline Indicador & $\begin{array}{l}\text { Curso } \\
16 / 17\end{array}$ & $\begin{array}{l}\text { Curso } \\
17 / 18\end{array}$ \\
\hline $\begin{array}{l}\text { Tasa de éxito (relación } \\
\text { porcentual entre el total de } \\
\text { créditos superados y el total de } \\
\text { créditos presentados) }\end{array}$ & $99,37 \%$ & $99,34 \%$ \\
\hline $\begin{array}{l}\text { Tasa de rendimiento } \\
\text { (relación porcentual entre el } \\
\text { total de créditos superados y el } \\
\text { número total de créditos } \\
\text { matriculados) }\end{array}$ & $64,96 \%$ & $75,44 \%$ \\
\hline
\end{tabular}

Tasa de abandono (del curso $\mathrm{X}$ : relación porcentual entre los estudiantes matriculados en el curso académico X-2, que no se han matriculado en los cursos X-1 y X)

En nuestra experiencia piloto, hemos analizado las mejoras obtenidas gracias a la implementación del EVEA, comparando los principales indicadores del título (ver Tabla 1) durante el curso 2016/17 (sin EVEA) con los obtenidos durante el curso 2017/18. Los datos referenciados pertenecen al grupo a distancia, debido a que la influencia del EVEA adquiere mayor notoriedad en este tipo de aprendizaje (los datos del grupo presencial son similares). Se han mejorado sensiblemente las tasas de rendimiento y de abandono. La tasa de éxito, muy positiva, apenas tiene variación.

Cabe aclarar que una tasa de abandono del 100\% (referente al curso 2016/17) no significa que el grupo se haya quedado sin estudiantes, se debe a que este indicador se calcula con datos de dos cursos anteriores al que se indica.

Respecto a la valoración del profesorado por parte del alumnado, no se observan diferencias significativas, en ambos cursos se obtiene un resultado alrededor de 4,5 sobre 5, estando por encima del valor medio del centro $(4,30)$ y de la universidad $(4,32)$ donde se imparte la docencia. Además en el informe del Docentia, en ambos cursos, la mayor parte del profesorado obtiene una calificación de excelente o notable.

Si consideramos la satisfacción del profesorado como instructor y como gestor del EVEA las valoraciones de ambos cursos son similares: planificación, coordinación, materiales (21,5 puntos sobre un máximo de 30$)$, desarrollo del proceso de aprendizaje (43,2 sobre 50), resultados del aprendizaje (15,9 sobre 20$)$, satisfacción global (80,5 sobre 100$)$.

\section{CONCLUSIONES}

La viabilidad de nuestra propuesta de implementación de un EVEA genérico, destinado a la enseñanza de asignaturas con contenido matemático, se ha constado en una experticia en el máster de matemática computacional que se ha referenciado. Se han implementado las acciones que se indican en Gregori et al. (2018), las cuales han facilitado la mejora del aprendizaje. Fundamentalmente se ha puesto énfasis en la tutorización personalizada, constante y activa para que el estudiante se sienta acompañado en su proceso de aprendizaje, con objeto de evitar el abandono de los estudios. Durante el curso 2017/18 se llevó a cabo la evaluación de dicho EVEA.

En general los resultados son muy positivos a la luz de los datos aportados. La disminución de la tasa de abandono resulta especialmente significativa en la mejora obtenida, comparándola con los datos aportados en Gregori et al. (2018). En dicho estudio se refiere que los cursos MOOC tienen una tasa de abandono alrededor del $90 \%$ y los cursos de la UNED alrededor del $50 \%$.

Aunque los datos de satisfacción son también muy positivos, pensamos que el análisis de un solo curso no es suficiente. Se debe considerar un periodo más largo e ir introduciendo los ajustes necesarios para eliminar las deficiencias detectadas. Los ajustes más significativos que hemos detectado que se deben considerar son seguir prestando especial atención a la 
tutorización, actualizar contenidos y adecuar la temporización de las tareas asignadas.

\section{AGRADECIMIENTOS}

Este trabajo ha sido parcialmente apoyado por la Unidad de Soporte Educativo de la Universitat Jaume I de Castelló (proyectos: 3595/18, 3596/18, 3598/18).

\section{REFERENCIAS}

Arnal, A., Castelló, J., Galindo, C., Gregori, P., Martínez, V. y Momparler, J. (2016). Learning Outcomes And Competencies In Mathematics After A Four Year Experience In Engineering Degrees. INTED 2016.

Arnal, A., Galindo, C., Gregori, P. y Martínez, V. (2017). Empowering Face-To-Face Learning With Online Learning At Undergraduate Level. INTED 2017.

Castañeda, J. (2016). Movilidad Virtual en el Máster en Matemática Computacional (Tesis Doctoral). Universitat Jaume I, Castelló.

García Aretio, L. (2008). NetACTIVE. UNED Madrid.

Gil, A. y Roca-Piera, J. (2015, julio, 14). Movilidad virtual, reto del aprendizaje de la Educación Superior en la Europa
2020. Revista de Educación a Distancia, (26). Recuperado de http://www.um.es/ead/red/26/.

Gregori, P., Martínez, V. y Moyano-Fernández, J. J. (2018). Basic actions to reduce dropout rates in distance learning, Evaluation and Program Planning, Vol. 66, pp. 48-52. doi: $10.1016 /$ j.evalprogplan.2017.10.004

Martínez, V. (2017). Educación presencial versus educación a distancia. La Cuestión Universitaria, Vol. 9, pp. 108-116. Recuperado de http://polired.upm.es/index.php/lacuestionuniversitaria/a rticle/view/3582/3662

Pitarch, A. M., Sáez, B., Gallego, T., Huedo, P., García, J. A. y Reig, L. (2013). Coordinación entre asignaturas en la docencia por proyectos en el grado en Arquitectura Técnica de la Universitat Jaume I. IV Jornada Nacional sobre Estudios Universitarios. Castellón, España: Publicacions de la Universitat Jaume I, pp.158-169.

Sein-Echaluce, M. L., Fidalgo, A. \& García-Peñalvo, F. (2015). Metodología de enseñanza inversa apoyada en b-learning y gestión del conocimiento. III Congreso Internacional sobre Aprendizaje, Innovación y Competitividad (CINAIC 2015). Madrid, España: Fundación General de la Universidad Politécnica de Madrid. 464-468. 